\title{
Effects of density-dependent dispersal behaviours on the speed and spatial patterns of range expansion in predator-prey metapopulations
}

\author{
A. Ramanantoanina ${ }^{\mathrm{a}, \mathrm{b}, *}, \mathrm{C} . \mathrm{Hui}^{\mathrm{c}}, \mathrm{A}$. Ouhinou ${ }^{\mathrm{a}, \mathrm{b}}$ \\ a Department of Mathematical Sciences, Stellenbosch University, Private Bag X1, Matieland 7602, South Africa \\ b African Institute for Mathematical Sciences, 6 Melrose Road, Muizenberg 7945, South Africa \\ ' Centre for Invasion Biology, Department of Botany and Zoology, Private Bag X1, Matieland 7602, South Africa
}

\section{A R T I C L E I N F O}

\section{Article history:}

Received 30 March 2011

Received in revised form 6 August 2011

Accepted 11 August 2011

\section{Keywords:}

Prey evasion

Predator pursuit

Rate of spread

Spatial synchrony

Metapopulation persistence

\begin{abstract}
A B S T R A C T
Dispersal can strongly affect the spatiotemporal dynamics of a species (its spread, spatial distribution and persistence). We investigated how two dispersal behaviours, namely prey evasion (PE) and predator pursuit (PP), affect the dynamics of a predator-prey system. PE portrays the tendency of prey avoiding predators by dispersing into adjacent patches with fewer predators, while PP describes the tendency of predators to pursue the prey by moving into patches with more prey. Based on the Beddington predation model, a spatially explicit metapopulation model was built to incorporate PE and PP. Numerical simulations were run to investigate the effects of $\mathrm{PE}$ and $\mathrm{PP}$ on the rate of spread, spatial synchrony and the persistence of populations. Results show that both PE and PP can alter spatial synchrony although PP has a weaker desynchronising effect than PE. The predator-prey system without PE and PP expanded in circular waves. The effect of PE can push the prey to distribute in a circular ring front, whereas the effect of PP can change the circular waves to anisotropic expansion. Furthermore, weak PE and PP can accelerate the spread of prey while strong and disproportionate intensities slow down the range expansion. The effects of PE and PP further enhance the population size, break down the spatial synchrony and promote the persistence of populations.
\end{abstract}

(C) 2011 Elsevier B.V. All rights reserved.

\section{Introduction}

Predation is one of the most fundamental interspecific interactions in ecology. Early theories regarding the predator-prey systems often implicitly assume well-mixed homogenous populations in space, namely the mean-field assumption (e.g. Rosenzweig, 1963; Beddington et al., 1975; Berryman, 1992) and thus violate the reality that the spatial distribution of species is rarely homogenous (Kokubun et al., 2008) but spatial autocorrelated (Fortin and Dale, 2005; Hui et al., 2010). Furthermore, the conventional non-spatial models do not allow the implementation of different survival strategies that can lead to the spatial heterogeneity of species distribution. As such, spatial predation models have been developed to mainly examine the effect of spatially explicit processes on the dynamics and viability of populations, such as models using partial differential equations, coupled ordinary differential equations,

\footnotetext{
* Corresponding author at: African Institute for Mathimatical Sciences 6 Melrose Road, Muizenberg 7945, Cape Town, South Africa.

E-mail address: ar@aims.ac.za (A. Ramanantoanina).
}

integrodifference equations and lattice models (e.g. Neubert et al., 1995; Murray, 2001; Petrovskii, 2006).

One important process that can substantially affect the spatiotemporal dynamics is dispersal (as species' behaviour strategy; Londsale, 1993). Living organisms often display a variety of behaviour strategies in nature to enhance their survival (e.g. seeking refuge and forming swarms for anti-predation; Siegfried, 1975; Linden, 2007; Weng, 2007), where different dispersal (or movement) behaviours are bound to arise from the optimization of species evolutionary fitness. Consequently, different ways of capturing prey have been observed in predators. While some predators sit and wait for their prey at hidden places (e.g. crab spiders; Morse, 2006), others actively change their hunting ground according to prey density (e.g. pythons; Madsen, 1996). In contrast, prey can also improve its survival rate by actively avoiding encountering potential predators (e.g. white sharks; Weng, 2007). Besides affecting its own survival, dispersal behaviours can also potentially affect the invasiveness and spread of non-native species (Holway and Suarez, 1999; Rehage and Sih, 2004). However, it is still unclear how species dispersal behaviours affect the speed and spatial patterns of species range expansion, especially in a predator-prey system. 
Depending on the causes and modes of dispersal, the spatial patterns exhibited can vary from spatial synchrony (i.e. the dynamics of populations at different localities coincide) to spatial chaos ( $\mathrm{Li}$ et al., 2005). While the spatial heterogeneity of species distribution enhances the survival by providing refuge and thus promoting rescue effect (Allen et al., 1993; Bommarco et al., 2007), spatial synchrony tends to be detrimental to the persistence of metapopulations (Matter, 2001). Studies of this kind mainly focus on (i) the effect of predator's dispersal behaviour (that depends on prey density) on the dynamics of the system (Chakraborty et al., 2007; El Abdlaoui et al., 2007; Ainseba et al., 2008; Tao, 2010) and (ii) the effects of prey refuge and density-dependent mortality on species persistence (Gonzalez-Olivares and Ramos-Jiliberto, 2003; Forrester and Steele, 2004). An emerging question from invasion biology is how such dispersal behaviours affect the rate of spread of non-native species in novel environments (Okubu, 1986; Shigesada and Kawasaki, 1997; Sutherland et al., 2002). Closely relevant to the monitoring and control of invasive species, it is only recently that this question has started to receive attention (e.g. Tsyganov et al., 2004).

We here explore the effect of two density-dependent dispersal behaviours, namely prey evasion (PE) and predator pursuit (PP), on the spatiotemporal dynamics of a predator-prey using a discrete metapopulation model. Prey evasion (PE) describes the behaviour of predator avoidance in prey - that is, animals often avoid encountering their predators by actively fleeing to places with lower predator density. Predator pursuit (PP), on the other hand, portrays the tendency of predators to pursuit prey by moving from their current location to high-prey density areas (Tsyganov et al., 2004). The effects of PE and PP have been investigated in a semidiscrete framework (Li et al., 2005), showing that these dispersal behaviours can alter the spatial synchrony in a predator-prey system and promote population persistence.

Our model is based on the model published by Beddington et al. (1975) and adapted to incorporate PE and PP. A similar model was used by McCann et al. (2000) for building a three-patch model to investigate the outbreak of populations in a discrete system. Our metapopulation model consists of predator-prey systems in different habitat patches which are spatially linked by nonlinear density-dependent dispersals. We focus on the effects of PE and PP on the metapopulation persistence and the rate of spread when a species expands its range, both of which are of crucial importance for controlling the spread of invasive species and conserving endangered species.

\section{Models}

\subsection{Dispersal-reproduction model}

We assume a dispersal-growth model, that is, the model consists of two phases. During the dispersal phase, a fraction of prey and predators move from their location to more suitable surrounding patches according to density-dependent dispersals, namely dispersal due to random walk, PE and PP. During the growth phase, populations within a patch undergo growth and experience predation. In the following, we let $N_{i, t}$ and $P_{i, t}$ denote the density of the prey and predator populations in the patch $i$ at time $t$, respectively.

The dispersal phase includes three components: random-walk, PP and PE. Firstly, individuals undergo random-walk which leads to individuals diffusing from crowded patches to less-crowded adjacent patches. We assumed that the patches were homogeneous and that the prey and predator had the same sighting range so that individuals can compare equal amount of neighbouring patches. The net number of prey individuals $R W\left(N_{i, t}\right)$ gained by patch $i$ due to random walk between the patch and its neighbouring patches can be depicted as follows:

$R W\left(N_{i, t}\right)=\frac{1}{\# \zeta_{i}} \sum_{j \in \zeta_{i}}\left(\frac{\max \left(0, N_{j, t}-N_{i, t}\right)}{N_{i, t}+N_{j, t}} N_{j, t}-\frac{\max \left(0, N_{i, t}-N_{j, t}\right)}{N_{i, t}+N_{j, t}} N_{i, t}\right)$

where $\zeta_{i}$ designates the set of patches surrounding the patch $i$, and $\# \zeta_{i}$ is the number of patches surrounding the patch $i$. We used the Moore neighbourhood which consisted of all patches sharing an edge or corners with the patch. The random walk of the predator species was defined analogously.

Secondly, the movement of individuals between cells (patches) can also be affected by the two dispersal behaviours (i.e. PE and PP). We modified the form of PE and PP previously presented by McCann et al. (2000) and Li et al. (2005) to ensure the solutions are positive, and derived the following revised forms for the net number of prey gained in patch $i\left(P E_{i}\right)$ due to the effect of $P E$ (i.e. the number of prey fled to patch $i$ from its neighbouring patches minus the number of prey fled from patch $i$ to its neighbouring patches):

$P E_{i}=\frac{1}{\# \zeta_{i}} \sum_{j \in \zeta_{i}}\left(\frac{\max \left(0, P_{j, t}-P_{i, t}\right)}{P_{i, t}+P_{j, t}} N_{j, t}-\frac{\max \left(0, P_{i, t}-P_{j, t}\right)}{P_{i, t}+P_{j, t}} N_{i, t}\right)$

Similarly, we can define the net number of predators gained in patch $i$ due to the effect of PP (i.e. the number of predators following prey from neighbouring patch to patch $i$ minus the number of predators following prey from patch $i$ to its neighbouring patch):

$P P_{i}=\frac{1}{\# \zeta_{i}} \sum_{j \in \zeta_{i}}\left(\frac{\max \left(0, N_{i, t}-N_{j, t}\right)}{N_{i, t}+N_{j, t}} P_{j, t}-\frac{\max \left(0, N_{j, t}-N_{i, t}\right)}{N_{i, t}+N_{j, t}} P_{i, t}\right)$

Let $v$ and $\beta$ denote the intensity of PE and PP, respectively, $\mu$ and $\alpha$ the maximum dispersal rate of the prey and predators, and $\tilde{N}_{i, t}$ and $\tilde{P}_{i, t}$ the post-dispersal prey and predator population densities of patch $i$ at time $t$, respectively. We thus have the population dynamics for the dispersal phase:

$\tilde{N}_{i, t}=N_{i, t}+\frac{\mu}{1+v}\left(R W\left(N_{i, t}\right)+v P E_{i}\right)$
$\tilde{P}_{i, t}=P_{i, t}+\frac{\alpha}{1+\beta}\left(R W\left(P_{i, t}\right)+\beta P P_{i}\right)$

The coefficients of the dispersal behaviours of the prey were chosen such that (i) when the PE intensity $v$ is negligible, in the absence of predators for instance, the dispersal of prey is driven completely by random walk with a dispersal rate $\mu$, (ii) when $v$ is large, the dispersal of prey is dominated by PE with the same dispersal rate $\mu$. The coefficients of the dispersal of predators were defined analogously.

The reproduction phase includes population growth and predation (Beddington et al., 1975), adjusted to incorporate the above dispersal phase:

$N_{i, t+1}=\tilde{N}_{i, t} e b\left(1-\frac{\tilde{N}_{i, t}}{K}\right)-a \tilde{P}_{i, t}$

$P_{i, t+1}=c \tilde{N}_{i, t}\left(1-e-a \tilde{P}_{i, t}\right)$

with positive initial distribution, $N_{i, 0} \geq 0, P_{i, 0} \geq 0, i=0,1, \ldots n$, where $b$ is the intrinsic growth rate of prey, $K$ is the carrying capacity of prey per patch, $a$ is the predator's attack rate and $c$ is the efficiency of conversion of prey into predators. The population flow is shown in Fig. 1 when $N_{1, t}>N_{2, t}$ and $P_{1, t}<P_{2, t}$. In the figure, PE and PP occur from the first patch to the second one. A summary of the parameters and variables used in this model is given in Table 1 . It is worth noting that with positive initial population density, the solution to the above model remains positive and is bounded for all time $t$. 


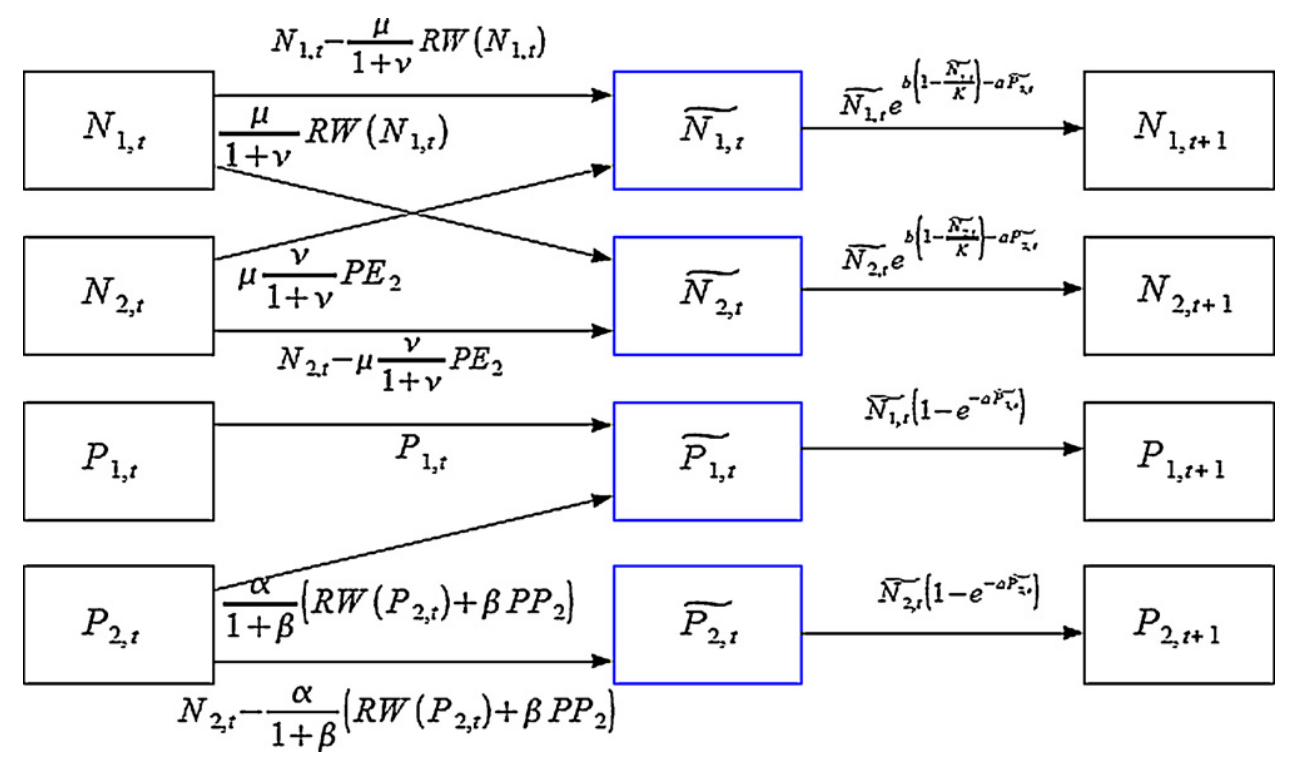

Fig. 1. A step of the two-patch model. Fluxes are shown for $N_{1, t}>N_{2, t}$ and $P_{1, t}<P_{2, t}$ in the illustration.

Table 1

Descriptions of parameters and variables.

\begin{tabular}{ll}
\hline Variable/parameter & Description \\
\hline$N_{i, t}$ & Prey population size before migration in patch $i$ at time $t$. \\
$\tilde{N}_{i, t}$ & Prey population size after migration in patch $i$ at time $t$ \\
$P_{i, t}$ & Predator population size before migration in patch $i$ at \\
& time $t$ \\
$\tilde{P}_{i, t}$ & Predator population size after migration in patch $i$ at \\
& time $t$ \\
$P E_{i}$ & Prey evasion between a patch $i$ and its neighbours \\
$v$ & Intensity of PE \\
$P P_{i}$ & Predator pursuit between a patch $i$ and its neighbours \\
$\beta$ & Intensity of PP \\
$\mu$ & Maximum migration rate of preys \\
$\beta$ & Maximum migration rate of predators \\
$a$ & Predators attack rate \\
$c$ & Conversion efficiency of preys into predators \\
$K$ & Prey's carrying capacity per patch \\
$b$ & Prey's intrinsic growth rate \\
$\zeta_{i}$ & Set of neighbours of a patch $i$ \\
$\# \zeta_{i}$ & Number of neighbours of a patch $i$ \\
\hline
\end{tabular}

\subsection{Numerical simulations}

In most cases of the simulation, we used the same set of parameters ( $b=0.5, c=1, a=0.5, K=15, \mu=0.75$ and $\alpha=0.75$ ) but only changed the intensity of PE and PP (i.e. $v$ and $\beta$ ) to investigate their effects on the spatiotemporal dynamics of the predator-prey system. Both the prey and the predators were initially located in the centre of the lattice, leaving other patches empty. Reflective boundaries were used (i.e. individuals move out of the lattice will be located back to their pre-dispersal locations). We studied the effects of different levels of PE and PP on the metapopulation persistence, the spatiotemporal dynamics of the predator-prey metapopulations, specifically, spatial synchrony, and the rate of spread.

To investigate the effects of PE and PP on the persistence of the metapopulation, we started by exploring the effects of PE and PP on the stability of a two-patch model. We then undertook extensive numerical simulations to investigate the effects of PE and PP on the persistence of the metapopulation on a large lattice. The persistence of the metapopulation was measured by the mean population density over the occupied patches. Non-symmetric steady-states which may arise in large lattices were not captured in the study.
Spatial synchrony depicts the coincidence in the temporal fluctuations in local population size, and can be measured using a method, based on change, described by Buonaccorsi (2001). For two patches $i$ and $j$, the level of synchrony is given by

$s_{i j}=2 \frac{A_{i j}}{t-1}-1$

where $A_{i j}$ is the number of times that the population size in the patches $i$ and $j$ fluctuate in the same direction. The level of synchrony for the lattice was then defined as the mean synchrony of all pairs of patches in the lattice.

To calculate the rate of spread, we adopted the method used by Kawasaki and Shigesada (2007) for a discrete-time onedimensional model to the two-dimensional lattice. The rate of spread is given by

$r(t)=\frac{1}{t} \sum_{\tau=1}^{t}(R(\tau)-R(\tau-1))=\frac{R(t)-R(0)}{t}, \quad t>0$

where $R(t)$ is the approximated radius occupied by the species at time $t$. Here, "occupied patch" refers to a patch with a population size higher than a threshold of detection $\sigma$. If we denote the area occupied by the species, that is also the number of occupied patches, by $S(t)$, then the approximated radius is given by

$R(t)=\sqrt{\frac{S(t)}{\pi}}$

\section{Results}

\subsection{Asymptotic behaviour}

The two-patch model has three steady states, including a trivial steady state $\left(N_{1}=0, N_{2}=0, P_{1}=0, P_{2}=0\right)$, a semi-trivial steady state $(K, K, 0,0)$ and a non-trivial steady state $\left(N^{*}, N^{*}, P^{*}, P^{*}\right)$ where $N$ is a solution of

$$
\frac{b\left(1-\left(N^{*} / K\right)\right)}{a N^{*}}=1-e^{-b\left(1-\left(N^{*} / K\right)\right.}
$$

with $0<N^{*}<K$ and $P^{*}$ is given by

$P^{*}=\frac{b}{a}\left(1-\frac{N^{*}}{K}\right)$ 

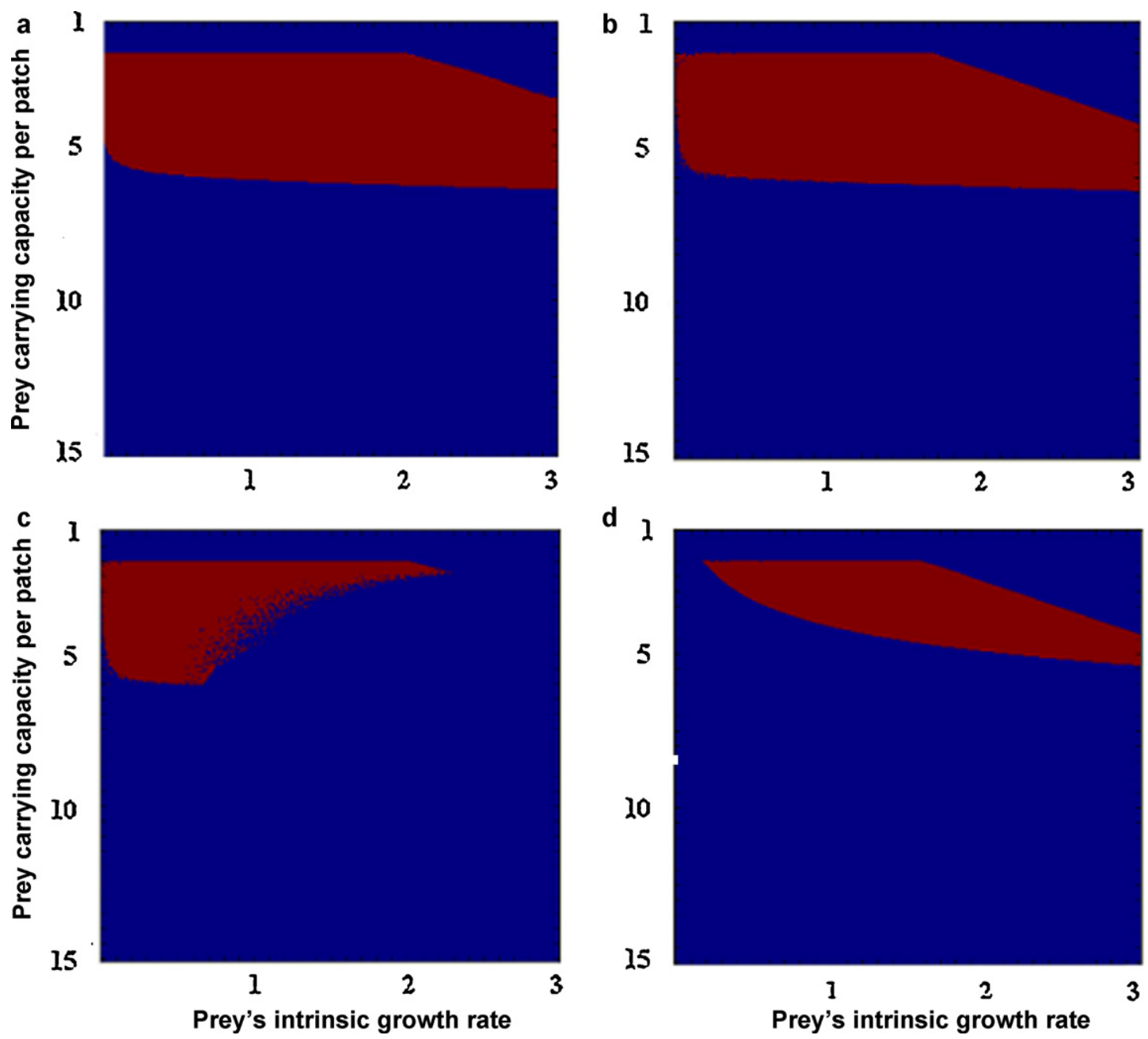

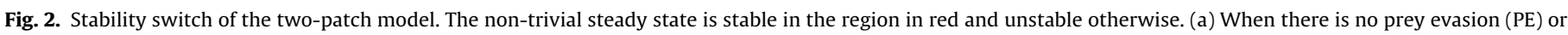
predator pursuit (PP). (b) Strong PE $(v=30)$ but no PP. (c) Strong PP $(\beta=30)$ but no PE. (d) Strong PE and PP, with $v=\beta=30$.

The trivial steady state is stable regardless of the parameter values. The stability region of the non-trivial steady state became largest in the parameter plane when there was no PE and PP in the system (Fig. 2). The population started to oscillate for high prey carrying capacity $(K)$, and the predators became extinct for low prey carrying capacity and prey intrinsic growth rate $(b)$. The stability region of the non-trivial steady state shrank for high prey intrinsic growth rate, driving the system to oscillate. The asymptotic mean population size of the prey was shown in Fig. 3 for $K=4$ and $b=0.5$, representing a stable non-trivial steady state(Fig. 2). The mean population size is low when PE and PP are small, and strong PE and PP lead to high population size. However, the effect of PP on the mean population size is much less than the effect of PE.

\subsection{Spatial distribution and rate of spread}

Spatial distributions of prey after 120 time steps were presented under different intensities of PE and PP (Fig. 4). When PE and PP were weak, the prey population expanded in circular waves and distributed evenly over the occupied patches. This uniform distribution of the population was gradually deteriorated as we increased the intensities of PE and PP either independently or simultaneously. When the intensity of PE was disproportionally higher than the intensity of PP, the prey population propagated in circular waves and accumulated in a circular ring front. When PP was stronger than PE, directional (anisotropic) expansions of the prey appeared and gave rise to a heterogeneous but symmetric distribution of the population. When both PE and PP were strong, the circular ring front associated with a strong PE became thinner

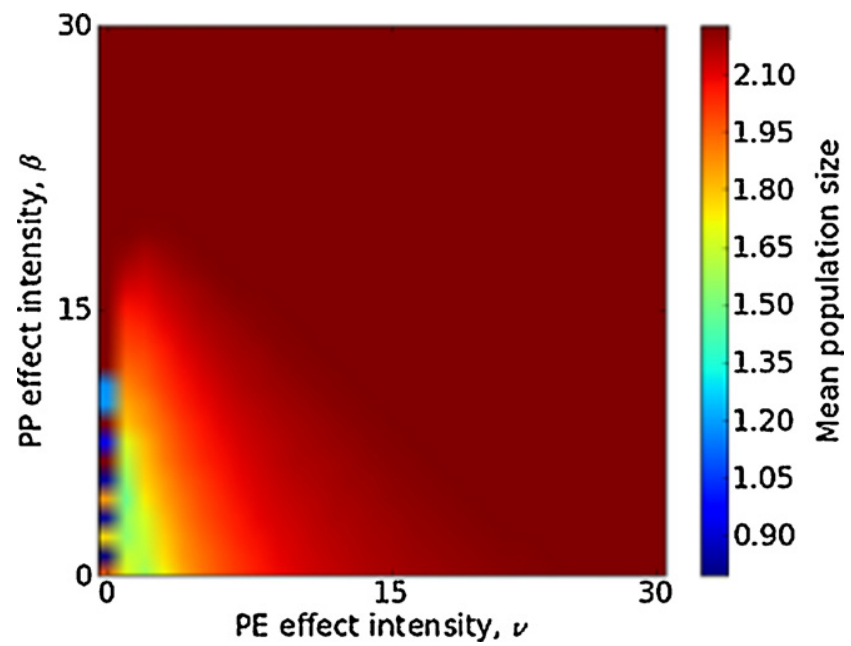

Fig. 3. A parameter plane showing the mean prey population size as a function of density-dependent prey evasion (PE) and predator pursuit (PP). Parameter values are set as $K=4, b=0.5, a=0.5, \mu=0.75, \beta=0.75$. PE and PP are increased in steps of 1 from 0 to 30 . 


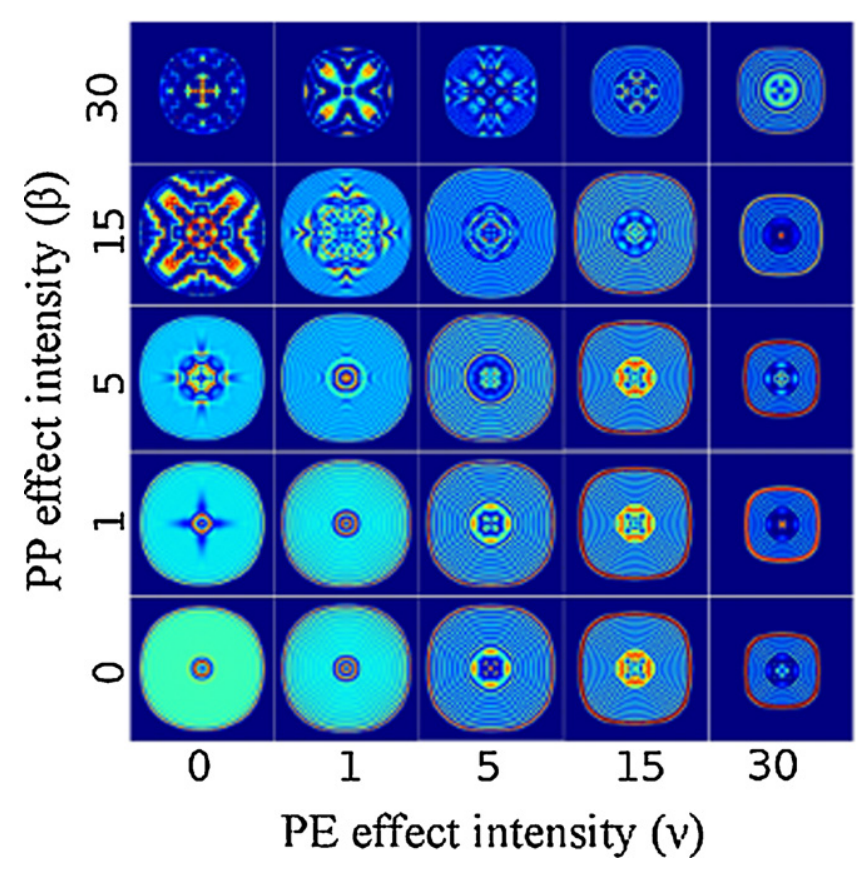

Fig. 4. Spatial distribution of the prey population after 120 generations on a $250 \times 250$ lattice. Parameter values are set as $b=0.5, c=1, a=0.5, K=15, \mu=0.75$ and $\alpha=0.75$. In the patterns, red refers to high population size and dark blue reflects low population size.

and the directional expansion associated with a strong PP became more isotropic.

The spatial synchrony of the prey was presented as a function of the intensities of PE and PP (Fig. 5), showing an asynchronous behaviour of populations in the lattice. Highly synchronized dynamics was observed for weak PE and PP. Both the PE and PP had a desynchronizing effect on the spatial dynamics. Furthermore, the level of synchrony was more sensitive to PE than PP. The spatial synchrony remained with the increase of PP intensity $(\beta)$ only, whereas the synchrony declined with the increase of PE intensity $(u)$. The least synchrony was reached for strong PE and PP simultaneously.

The rates of spread of the prey and predators were calculated according to the radius at the 120 times step with a threshold of

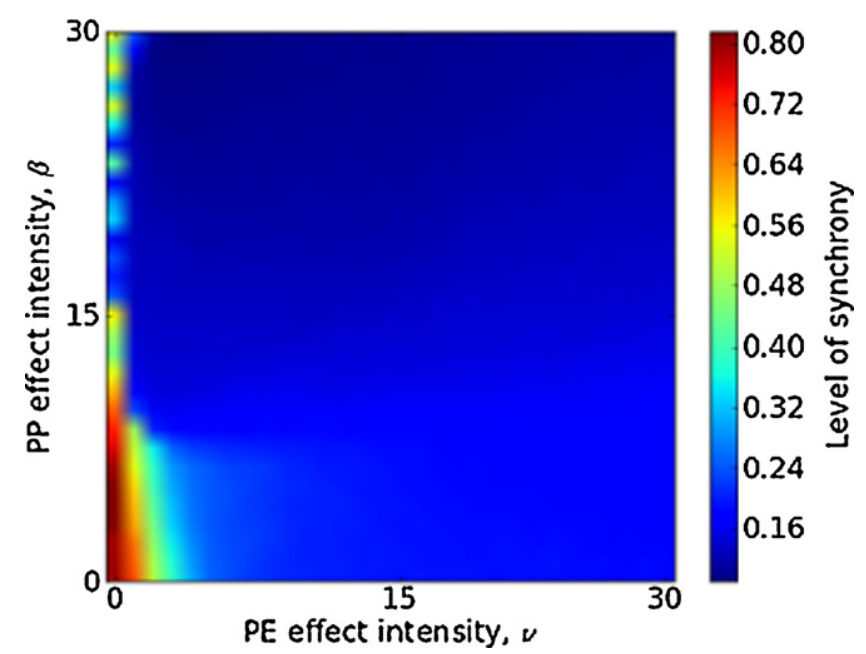

Fig. 5. A parameter plane showing the level of spatial synchrony of the prey population as a function of prey evasion and predator pursuit, calculated on a $20 \times 20$ lattice. Parameter values are set as $K=4, b=0.5, a=0.5, \mu=0.75, \alpha=0.75$. PE and PP are increased from 0 to 30 in steps of 1. detection of 0.01 (Fig. 6). The rate of spread for prey was correlated with the rate of spread for predators. The range expansion became faster with the increase of PE intensity but only when the PE intensity was below a certain threshold $\left(v^{*}(\beta)\right)$, beyond which the rate of spread started to decline with the PE intensity $(u)$. Similarly, the rate of spread was an increasing function of the PP intensity when $\beta<\beta^{*}(v)$, beyond which the rate of spread became a decreasing function of the PP intensity.

\section{Discussion}

\subsection{Dispersal behaviour and spatial distribution}

Spatial synchrony in population dynamics is mainly caused by three factors - (i) spatial autocorrelation inherent in the environment (Moran, 1953; Gao, 2007), (ii) interspecific density regulation through predation and parasitism (Ims, 2000; Gonzalez-Olivares and Ramos-Jiliberto, 2003) and (iii) density-independent dispersal (Jansen, 2001). For a predator-prey system in particular, even a small dispersal rate can lead to synchrony if the environment is homogeneous (Jansen, 2001). In contrast, we studied the effect of two density-dependent dispersals (PE and PP) on the spatial synchrony of predator-prey systems. The spatial synchrony reached peaks when the dispersals were only motivated by random walk and reduced whenever PE and PP were present. This is consistent with the results from a two-patch semi-discrete model ( $\mathrm{Li}$ et al., 2005). Moreover, PP alone has a less desynchronizing effect than PE. When PE is strong, however, prey can move to 'refuge' patches with low predator densities and thus grow in abundance before being detected by predators. This allows the growth of prey in 'refuge' patches while the remaining prey in the original patch is being depleted by predators, inducing a spatial asynchrony between refuge and original patches. Moreover, the decline of synchrony caused by the density-dependent dispersals was more severe in predator-prey systems than in single-species systems (Munkmuller and Johst, 2008).

Numerical simulations on the $250 \times 250$ lattices further confirmed that density-dependent dispersal is a force of asynchrony: an even spatial distribution of the prey population was observed when the patches are connected only via density-independent random walk (Fig. 4), consistent with Nguyen-Huu's (2006) results. The prey with a strong PE is mainly driven by fear of predators and thus takes the minimal-effort evasion strategy (Oshanin et al., 2009). This potentially explains the high concentration of prey in the range front for prey when there is a high PE intensity (the red ring in Fig. 4). In contrast, predators with a strong PP ability can quickly move into patches with a high prey density and deplete the local prey while the prey population in other patches expands, giving rise to the anisotropic expansion (Fig. 4). When PE and PP are both strong, the minimal-effort evasion of prey is interfered with by the anisotropic expansion, leading to strong asynchrony and spatial chaos (Li et al., 2005).

\subsection{Dispersal behaviour and the rate of spread}

Elucidating the effect of biotic interactions on the spread of invasive species is important for mitigating the detrimental impact of biological invasions on the recipient ecosystems (Petit et al., 2008). The rate of spread has been shown to be more sensitive to longdistance dispersal (Lewis, 1997; Shigesada and Kawasaki, 1997). Predation, on the other hand, has been speculated to be able to slow down the expansion of a prey species (Owen, 2001). Our results suggest (i) that the velocity of range expansion for predators is closely tied with the velocity of prey range expansion (Fig. 6) and (ii) the rate of spread of both species is an increasing function of 

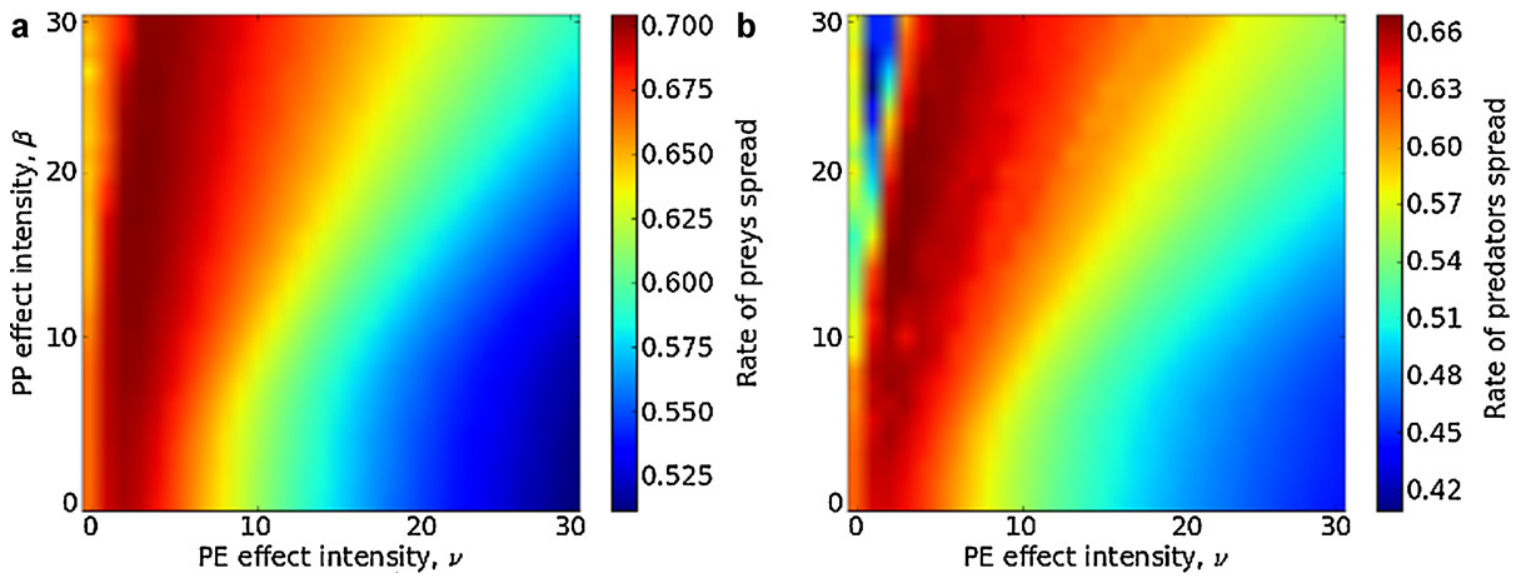

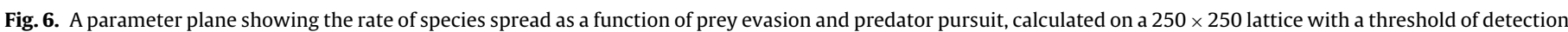

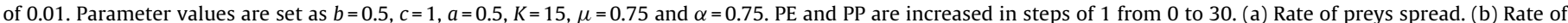
predators spread.

weak PE and PP but a decreasing function of strong PE and PP. The results are consistent with the results from Tsyganov et al. (2004) for a continuous one-dimensional model with weak PE and PP. When PE becomes more dominant (compared to PP and random walk), however, the prey avoid mainly predator-crowded areas. This implies a lack of prey for the predators and slows down the range expansion of the species. This further reduces the movement of the prey once the prey passed the area occupied by the predators, and slows down the range expansion (Fig. 6). On the other hand, when the PP is strong, the pursuiting success of predators is improved, which increases the chance of encountering prey. This will promote predation success and suppress the prey population from rapid range expansion. Therefore, density-dependent dispersal behaviour is indeed a factor that can change the speed of range expansion (Owen, 2001; Petrovskii, 2006).

\subsection{Dispersal behaviour and metapopulation persistence}

The idea that dispersal between different patches may favor the persistence of metapopulations has its origin in the 1990s. Predator-prey systems, where in particular non-spatial models tend to be unstable or lead to population extinction (Taylor, 1990), have been shown to be able to persist spatially even if local populations in different patches fluctuate (Wilson et al., 1993). In our case, the coexistence of the prey and predators was strongly mediated by the density-dependent dispersal behaviours of PE and PP. The stability diagram in Fig. 4 shows that, for the two-patch model, the prey and predators can stably coexist when the carrying capacity of the prey is low and when the PE and PP are weak. This stable coexistence was disturbed by the introduction of the density-dependent dispersal behaviours (PE and PP). For instance, an increase in the PE intensity could lead to the extinction of predators when the prey carrying capacity is low. This is because PE can affect the prey population by providing local refuges and thus induce a lack of prey for the predators.

Beside their effects on the asymptotic behaviour of the population dynamics, PE and PP can also influence the population size. Low population size can be expected when the patches are connected only by random-walk. Although prey does not go extinct in the system, small prey population size was indeed observed when only PP was present in the model. This is expected since the pursuit of predators can bring the system to a spatially evenly distribution in the absence of PE and PP. However, the mean population increased whenever PE was present in the model. The evasiveness of the prey does not only save a local prey population from predation, but also promotes the rescue effect between prey populations. Furthermore, an increase in the population size is also associated with a decrease in the level of spatial synchrony (Figs. 3 and 5), consistent with the idea that spatial synchrony reduces the persistence of the population (Lloyd and May, 1999; Matter, 2001).

Overall, our findings suggest that the two density-dependent dispersal behaviours, $\mathrm{PE}$ and $\mathrm{PP}$, can provoke asynchronous dynamics even in the absence of environmental heterogeneity, and thus both can improve the metapopulation persistence. Specifically, PE has a stronger desynchronizing effect on the dynamics than PP. Furthermore, weak density-dependent dispersal behaviours promote the spread of the prey and predator. However, when one of the two density-dependent dispersal PE and PP is strong, the range expansion will slow down. These results provide theoretical clues for reducing the rate of spread of problematic invasive species by choosing appropriate biological control agents that can provoke strong density-dependent dispersal of either the agent or targeted species.

\section{Acknowledgments}

This work was supported by the NRF Blue Skies Programme, Subcommittee B fund at the University of Stellenbosch, DST-NRF Centre of Excellence for Invasion Biology and the African Institute for Mathematical Sciences.

\section{References}

Ainseba, B.E., Bendahmane, M., Noussair, A., 2008. A reaction-diffusion system modelling predator-prey with prey taxis. Nonlinear Analysis: Real World Application 9, 2086-2105.

Allen, J.C., Schaffer, W.M., Rosko, D., 1993. Chaos reduces species extinction by amplifying local population noise. Nature 364, 229-232.

Beddington, J.R., Free, C.A., Lawton, J.H., 1975. Dynamic complexity in predator-prey models framed in difference equations. Nature 225, 58-60.

Berryman, A.A., 1992. The origins and evolution of predator-prey theory. Ecology 73, 1530-1535.

Bommarco, R., Firle, S., Ekbom, B., 2007. Outbreak suppression by predators depends on spatial distribution of prey. Ecological Modelling 201, 163-170.

Buonaccorsi, J.P., 2001. Measuring and testing for spatial synchrony. Ecology 82, 1668-1679.

Chakraborty, A., Singh, M., Lucy, D., Ridland, P., 2007. Predator-prey model with prey-taxis and diffusion. Mathematical and Computer Modelling 46, 482-498.

El Abdlaoui, A., Auger, P., Kooi, B.W., Bravo de la Parra, R., Mchich, R., 2007. Effects of density dependent migrations on stability of two-patch predator-prey model. Mathematical Biosciences 210, 335-354.

Forrester, G.E., Steele, M.A., 2004. Predators, prey refuges, and the scaling of densitydependent prey mortality. Ecology 85, 1332-1342. 
Fortin, M.J., Dale, M., 2005. Spatial Analysis: A Guide for Ecologists. Cambridge University Press.

Gao, M., 2007. Spatial synchrony in host-parasitoid populations. Ecological Modelling 204, 29-39.

Gonzalez-Olivares, E., Ramos-Jiliberto, R., 2003. Dynamics consequences of prey refuges in a simple model: more prey, fewer predators and enhanced stability. Ecological Modelling 166, 135-146.

Holway, D.A., Suarez, A.V., 1999. Animal behavior: an essential component of invasion biology. Trends in Ecology \& Evolution 14, 328-330.

Hui, C., Veldtman, R., McGeoch, M.A., 2010. Measures, perceptions and scaling patterns of aggregated species distributions. Ecography 33, 95-102.

Ims, R.A., 2000. Spatial synchronization of vole population dynamics by predatory birds. Nature 408, 194-196.

Jansen, V.A., 2001. The dynamics of two diffusively coupled predator-prey populations. Theoretical Population Biology 59, 119-131.

Kawasaki, K., Shigesada, N., 2007. An integrodifference model for biological invasions in a periodically fragmented environment. Japan Journal of Industrial Applied Mathematics 24, 3-15.

Kokubun, N., Iida, K., Mukai, T., 2008. Distribution of murres (Uria spp.) and their prey south of St. George Island in the southeaster Bering Sea during the summers of 2003-2005. Deep-Sea Research 55, 1827-1836.

Lewis, M.A., 1997. Variability, patchiness, and jump dispersal in the spread of an invading population. In: Tilman, D., Karieva, D. (Eds.), Spatial Ecology: The role of space in population dynamics and interspecific interactions. Monographs in population biology. , pp. 46-69.

Li, Z., Gao, M., Hui, C., Han, X., Shi, H., 2005. Impact of predator pursuit and prey evasion on synchrony and spatial in metapopulation. Ecological Modelling 185, $245-254$.

Linden, E., 2007. The more the merrier: swarming as an antipredator strategy in the mysid Neomysis integer. Aquatic Ecology 41, 299-307.

Lloyd, A.L., May, A.A., 1999. Synchronicity, chaos and population cycles: spatial coherence in an uncertain world. Trends in Ecology and Evolution 14, 417-418.

Londsale, W.M., 1993. Rates of spread of an invading species - Mimosa pigra in northern Australia. Journal of Ecology 81, 513-521.

Madsen, T.A., 1996. Seasonal migration of predators and prey - a study of pythons and rats in tropical Australia. Ecology 77, 149-156.

Matter, F.S., 2001. Synchrony, extinction, and dynamics of spatially segregated, heterogeneous populations. Ecological Modelling 141, 217-226.

McCann, K., Hastings, A., Harisson, S., Wilson, W., 2000. Population outbreaks in discrete world. Theoretical Population Biology 57, 97-108.

Moran, P., 1953. The statistical analysis of the canadian lynx cycle. Australian Journal of Zoology 1, 291-298.

Morse, D.H., 2006. Fine-scale substrate use by a small sit-and-wait predator. Behavioural Ecology 17, 405-409.
Munkmuller, T., Johst, K., 2008. Spatial synchrony through density independent versus density-dependent dispersal. Journal of Biological Dynamics 2 (1), 31-39. Murray, J.D., 2001. Mathematical Biology: I. An Introduction. Springer.

Neubert, M.G., Kot, M., Lewis, M.A., 1995. Dispersal and pattern formation in discrete-time predator-prey model. Theoretical Population Biology 48, 7-43.

Nguyen-Huu, T., 2006. Spatial synchrony in host-parasitoid models using aggregation of variables. Mathematical Bioscience 203, 204-221.

Okubu, A., 1986. Diffusion-type models for avian range expansion. In: Acta XIX Congressus Internationalis Ornithologici, vol. 1. University of Ottawa Press, pp. 1038-1049.

Oshanin, G., Vasiliyev, O., Krapivsky, P.L., Klafter, J., 2009. Survival of an evasive prey. PNAS 33, 13696-13701.

Owen, M.R., 2001. How predation can slow, stop or reverse a prey invasion. Bulletin of Mathematical Biology 63, 655-684.

Petit, J.N., Hoddle, M.S., Grandgirard, J., Roderick, G.K., Davies, N., 2008. Shortdistance dispersal behavior and establishment of the parasitoid Gonatocerus ashmeadi (Hymenoptera: Mymaridae) in Tahiti: Implications for its use as a biological control agent against Homalodisca vitripennis (Hemiptera: Cicadellidae) Biological Control 45, 344-352.

Petrovskii, S.V., 2006. Exactly Solvable Models of Biological Invasion. Chapman \& Hall/CRC.

Rehage, J.R., Sih, A., 2004. Dispersal behavior, boldness, and the link to invasiveness: a comparison of four Gambusia species. Biological Invasions 6, 379-391.

Rosenzweig, M.L., 1963. Graphical representation and stability conditions of predator-prey interactions. American Naturalist 47, 209-223.

Shigesada, N., Kawasaki, K., 1997. Biological Invasions: Theory and Practice. Oxford University Press.

Siegfried, W.R., 1975. Flocking as an anti-predator strategy in doves. Animal Behaviour 23, 504-508.

Sutherland, W.J., Gilland, J.A., Norris, K., 2002. Density-dependent dispersal in animals: concept, evidence, mechanisms and consequences. In: Bullock, J.M., Kenward, R.E., Hails, R.S. (Eds.), Dispersal Ecology. Blackwell, London, pp. 134-151.

Tao, Y., 2010. Global existence of classical solutions to a predator-prey mode with non-linear prey-taxis. Nonlinear Analysis: Real World Applications 11, 2056-2064.

Taylor, A.D., 1990. Metapopulations, dispersal, and predator-prey dynamics: an overview. Ecology 71, 429-433.

Tsyganov, M.A., Brindley, J., Holden, A., Biktashev, V.N., 2004. Soliton-like phenomena in one-dimensional cross-diffusion systems: a predator-prey pursuit and evasion example. Physica D 197, 18-33.

Weng, K.C., 2007. Migration and habitat of white sharks (Carcharodon carcharias) in the eastern Pacific Ocean. Marine Biology 152, 877-894.

Wilson, W.G., De Roos, A.M., McCauley, E., 1993. Spatial instabilities within the diffuse Lotka-Voltera system: individual based simulation result. Theoretical Population Biology 43, 91-127. 